\title{
Molecular architecture of a kinetochore-microtubule attachment site
}

\author{
Ajit P. Joglekar ${ }^{1}$, David C. Bouck ${ }^{1}$, Jeffrey N. Molk ${ }^{1}$, Kerry S. Bloom ${ }^{1}$ and Edward D. Salmon ${ }^{1,2}$
}

Kinetochore attachment to spindle microtubule plus-ends is necessary for accurate chromosome segregation during cell division in all eukaryotes. The centromeric DNA of each chromosome is linked to microtubule plus-ends by eight structural-protein complexes ${ }^{1-9}$. Knowing the copy number of each of these complexes at one kinetochore-microtubule attachment site is necessary to understand the molecular architecture of the complex, and to elucidate the mechanisms underlying kinetochore function. We have counted, with molecular accuracy, the number of structural protein complexes in a single kinetochore-microtubule attachment using quantitative fluorescence microscopy of GFP-tagged kinetochore proteins in the budding yeast Saccharomyces cerevisiae. We find that relative to the two Cse $4 \mathrm{p}$ molecules in the centromeric histone ${ }^{1}$, the copy number ranges from one or two for inner kinetochore proteins such as Mif2 $\mathrm{p}^{2}$, to 16 for the DAMDASH complex ${ }^{8,9}$ at the kinetochore- microtubule interface. These counts allow us to visualize the overall arrangement of a kinetochore-microtubule attachment. As most of the budding yeast kinetochore proteins have homologues in higher eukaryotes, including humans, this molecular arrangement is likely to be replicated in more complex kinetochores that have multiple microtubule attachments.

Accurate segregation of sister chromosomes during mitosis depends on the assembly of structural proteins at the kinetochore that link spindle microtubule plus-ends to centromeric DNA (CEN DNA). The structural arrangement of these proteins within the kinetochore underlies its function in force generation. It may also influence how the spindle assembly checkpoint senses kinetochore-microtubule attachment, and how errors in attachment are corrected to prevent chromosome mis-segregation. Although serial-section transmission electron microscopy has revealed the overall three-dimensional architecture of vertebrate kinetochores, the structure of individual kinetochore-microtubule attachment remains poorly characterized. Consequently, a mechanistic model of kinetochore function that integrates the details of its structure cannot currently be constructed. To understand the molecular architecture of a kinetochore-microtubule attachment site, we focused on counting the copy number for the core structural kinetochore proteins and protein complexes that are necessary for stable kinetochore-microtubule attachment.

Vertebrate kinetochores have a complex and dynamic structure with multiple attachment sites for kinetochore microtubules (about 20-25 in humans). In contrast, each kinetochore in budding yeast makes only one stable microtubule attachment during metaphase, suggesting that its molecular composition may be stable and making it an ideal organism for investigating kinetochore structure. There are more than 60 known kinetochore proteins in budding yeast and homologues for most of these proteins have been identified in vertebrate systems $\mathrm{s}^{10,11}$. Moreover, kinetochore function in metaphase chromosome alignment and segregation in anaphase is similar in budding yeast and higher eukaryotes ${ }^{12}$. Therefore, insight into the molecular architecture of the budding yeast kinetochore will be valuable for understanding the kinetochore-microtubule interaction in higher eukaryotes, including humans.

The DNA and protein composition of the budding-yeast kinetochore is well-understood. The kinetochore is built on a 125 base-pair stretch of CEN DNA that is composed of three distinct regions, CDE I, II and III. It is wrapped around a single nucleosome containing a centromerespecific histone $\mathrm{H} 3$ variant $^{1,13}$ named Cse4p (the human homologue is hsCENP-A). Proximal to this nucleosome is the CBF3 complex ${ }^{3}$ of four proteins that includes Ndc10p and Cep3p. The CBF3 complex specifically binds to the CDE III region of the CEN DNA. Another protein, Mif2p (hsCENP-C), binds to the CDE II region of CEN DNA. Next to this is the COMA complex of four proteins ${ }^{4}$ including Okp1p (hsCENPF) and Ctf19p. Recently, Spc105p was confirmed as a kinetochore protein $^{5}$. The homologue of Spc105p in Caenorhabditis elegans, KNL-1 (the uncharacterized human homologue is AF15q14), is necessary for microtubule attachment. Spc105p associates with the MIND complex ${ }^{14}$, which contains Mtw1p (hsMis12). The COMA complex also recruits the non-essential protein Ctf3p (hsCENP-I). In vertebrates, both CENP-I and Mis12 are required to recruit the outer kinetochore complex NDC80 (ref. 10). The NDC80 complex is a rod-like molecule that is approximately $50 \mathrm{~nm}$ long, and has globular ends ${ }^{7}$. It contains four proteins including Ndc80p (hsHec1) and Nuf2p (hsNuf2). Localization of antibodies to Ndc80 in vertebrate cells suggests that the Ndc80p-Nuf2p end

${ }^{1}$ Department of Biology, University of North Carolina, 607 Fordham hall, CB\# 3280, Chapel Hill, NC 27599, USA.

Correspondence should be addressed to E.D.S. (e-mail: tsalmon@email.unc.edu)

Received 20 February 2006; accepted 20 April 2006; published online 21 May 2006; DOI: 10.1038/ncb1414 


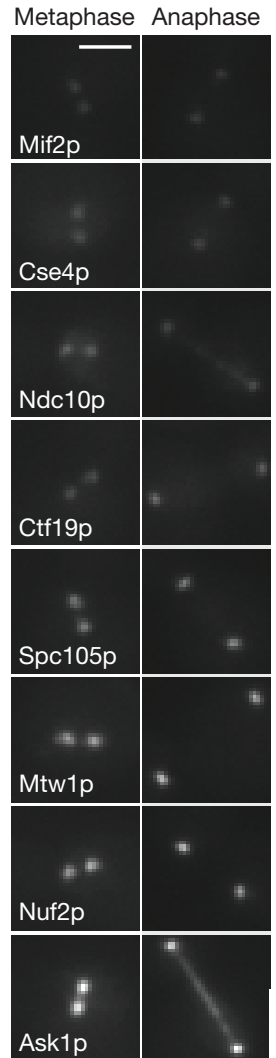

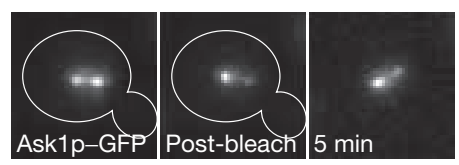
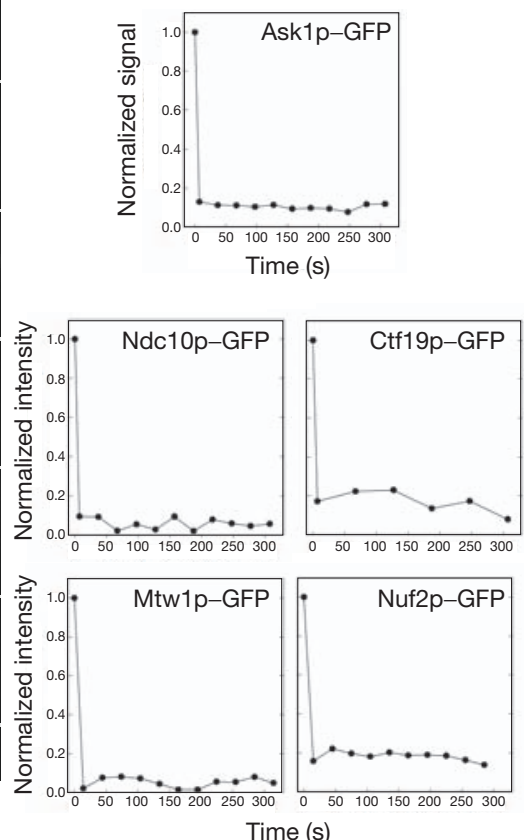

Time (s)

Figure 1 Localization and turnover of kinetochore proteins in metaphase and in anaphase-telophase. (a) Localization of representative GFP-tagged kinetochore proteins during metaphase and anaphase-telophase. At metaphase, the sister kinetochores become aligned on either side of the spindle equator into two distinct clusters each containing 16 kinetochores. Note that both CBF3 and the DAM-DASH complex also localize to the spindle in anaphase. The scale bar represents $2 \mu \mathrm{m}$. (b) Pre- and postphotobleaching images of a metaphase cell expressing Ask1p-GFP. Signal recovery (shown in the relative intensity versus time graphs) is undetectable for at least $300 \mathrm{~s}$. Recovery is similarly low for representative proteins from four other complexes.

of the NDC80 complex localizes proximal to the microtubule attachment site, whereas the other end localizes proximal to the inner centromere ${ }^{7,15}$. In budding yeast, the NDC80 complex and the microtubule associated protein complex, DAM-DASH, are both necessary for microtubule attachment ${ }^{10,11}$. The DAM-DASH complex is a heterodecamer and contains the protein Ask1p. Purified DAM-DASH complexes assemble into rings around microtubules in vitro ${ }^{8,9}$, which can slide passively along the microtubule lattice ${ }^{8}$.

There are important practical advantages in using budding yeast to obtain accurate protein counts using fluorescence microscopy. A protein of interest can be tagged at the carboxyl (C)-terminus with GFP at its chromosomal locus. The GFP-fluorescence signal is then a direct readout of the copy number for that protein ${ }^{16}$. The geometry of the budding-yeast spindle simplifies the quantification of the fluorescence signal from a GFP-tagged kinetochore protein. In metaphase, the sister kinetochores on the 16 chromosomes become arranged into two distinct clusters of sub-resolution size on either side of the spindle equator along the spindle axis (Fig. 1a). During anaphase, the kinetochore clusters move close to the spindle poles (anaphase A) as the spindle elongates (anaphase B) to push the poles apart (Fig. 1a). The core structural

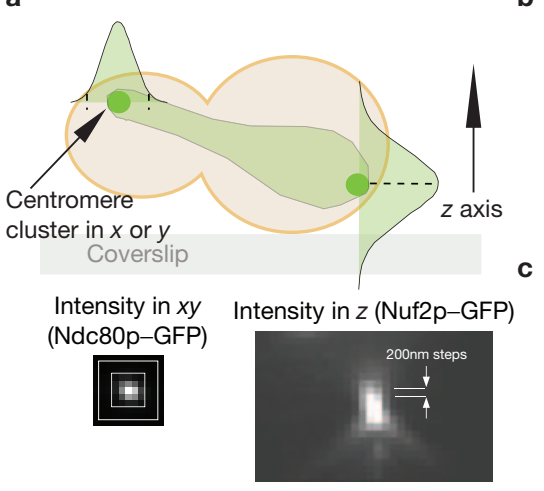

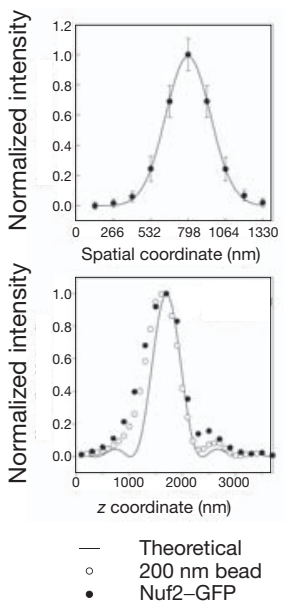

Figure $\mathbf{2}$ Characterization of the intensity distribution of a kinetochore cluster. (a) A schematic representation of a budding yeast cell expressing a GFP-tagged kinetochore protein in anaphase-telophase is shown. Signal was measured by integrating the signal intensity in the xy direction (shown for Ndc80p-GFP) in the plane that contains the maximum intensity pixel along the $z$ axis (shown for Nuf2p-GFP). (b) Fitting a Gaussian function to the anaphase-telophase intensity distribution in the xy plane for Nuf2p-GFP yields $\sigma=159 \mathrm{~nm}$ (s.d. for the Gaussian curve). Similar measurement for metaphase xy intensity distribution yields $\sigma=189 \mathrm{~nm}$. (c) The intensity distribution along the $z$ axis for a Nuf2p-GFP cluster and a $200 \mathrm{~nm}$ green fluorescent bead. The solid line represents the theoretical intensity distribution along the $z$ axis.

proteins are concentrated exclusively at kinetochores in metaphase and anaphase, with the exception of the CBF3 and DAM-DASH complexes. In anaphase, components of the latter two complexes partially dissociate from the kinetochore, and also associate with the spindle microtubules (Fig. 1a). Quantification of the fluorescence signal for a GFP-tagged protein in each kinetochore cluster provides the cumulative signal for 16 kinetochores, which can be used to measure the average number of molecules of that protein per kinetochore.

The centromeric histone Cse4p is a core component of the kinetochore. Cse $4 p$ shows virtually no turnover within a kinetochore cluster in metaphase, either through dissociation or through kinetochore movement from one spindle half to the other ${ }^{17}$. To test whether other structural kinetochore complexes are also similarly stable, fluorescence recovery after photobleaching (FRAP) was measured for representative GFP-tagged proteins from the CBF3, COMA, MIND, NDC80 and DAM-DASH complexes. One of the two sister kinetochore clusters was photobleached in both metaphase and anaphase cells (Fig. 1b, see Supplementary Information, Note 1 and Table S1). The recovery was found to be undetectably low $(<5 \%)$ in all cases. This low recovery is indicative of high protein stability at the kinetochore. FRAP measurements for CENP-I, CENP-H and Nuf2 in vertebrate cells show that these proteins are similarly stable $e^{18,19}$. These observations demonstrate that the core protein linkage between the CEN DNA and microtubule plus-ends is stable in metaphase and anaphase.

A comparative approach was used to count the number of molecules of a specific protein at the kinetochore. There are two Cse $4 p$ molecules in the centromeric nucleosome $e^{1,13}$ (see Supplementary Information, Note 2). The ratio of the average fluorescence signal for a kinetochore protein to the signal for Cse4p-GFP was multiplied by two to yield the average copy number per kinetochore for that protein. In each experiment, cells of two 
Table 1 Metaphase and anaphase-telophase ratio measurements

\begin{tabular}{|c|c|c|c|c|c|c|}
\hline Complex & Protein & Vertebrate homologue & Metaphase ratio & Anaphase ratio & Metaphase number & Anaphase number \\
\hline Nucleosome & Cse4p & hSCENP-A & 1 & 1 & 2 & 2 \\
\hline CBF3 & Ndc10p & - & $1.9 \pm 0.2$ & $1.3 \pm 0.01$ & 4 & $2-3$ \\
\hline - & Mif2p & hsCENP-C & $5.4 \pm 0.4 *$ & $5.5 \pm 0.10 *$ & $1-2$ & $1-2$ \\
\hline COMA & Ctf19p & hsCENP-F & $3.4 \pm 0.3^{*}$ & $3.4 \pm 0.20 *$ & 3 & 2 \\
\hline- & Spc105 & CeKNL-1 & $2.4 \pm 0.01$ & $2.4 \pm 0.01$ & 5 & 5 \\
\hline NDC80 & Nuf2p & hsNuf2 & $4.0 \pm 0.2$ & $3.6 \pm 0.20$ & 8 & 7 \\
\hline DAM-DASH & Ask1p & - & $9.0 \pm 1$ & $5.3 \pm 0.30$ & $16-20$ & $10-11$ \\
\hline CTF3 & Ctf3p & hsCENP-I & - & $0.5 \pm 0.01$ & - & 1 \\
\hline CHL4-IML3 & Chl4p & - & - & $0.26 \pm 0.01$ & - & $<1$ \\
\hline NKP1-NKP2 & Nkp2p & - & - & $6.1 \pm 0.05^{*}$ & - & 1 \\
\hline
\end{tabular}

The ratios shown are the average ratios obtained from three experiments with at least 20 measurements for metaphase cells, and at least two experiments with up to 80 measurements for late anaphase-telophase cells. The coefficient of variation (s.d. / mean) was better than 0.26 in all the measurements with the exception of Cep3p, for which the coefficient is 0.5 . The asterisks indicate that the reported ratio is (Nuf2p - signal) : (protein signal). Mif2p-GFP, Ctf19p-GFP and Nkp2-GFP measurements were carried out with Nuf2p-GFP as the reference signal (see Methods).

different strains - one expressing a GFP-tagged protein of interest and the other expressing Cse4p-GFP - were mixed together and immobilized on a coverslip for imaging (see Methods). An image stack, with a $200 \mathrm{~nm}$ separation along the $z$ axis between consecutive images, was then obtained for each cell (Fig. 2a). The maxima of the intensity distribution of each kinetochore cluster along the $z$ axis was determined with an average underestimation of $4 \%$ (Fig. 2c, see Supplementary Information, Note 3). At this maxima, the fluorescence was integrated over a $5 \times 5$ pixel (for anaphase-telophase cells) or $6 \times 6$ pixel (for metaphase cells) region. The dimensions of the signal and the background region are dictated by the spread of the fluorescence intensity for a kinetochore cluster, which can be characterized by fitting the spatial intensity distribution with a Gaussian curve (Fig. 2b, see Supplementary Information, Note 4). The total signal was then obtained by integration over the intensity distribution using the $2 \sigma$ limit (where $\sigma=$ s.d. for the Gaussian curve), after subtracting the background signal. Obtaining the ratio of the average signal values for the GFP-tagged protein of interest and Cse4p-GFP in each experiment avoids a direct evaluation of the in vivo fluorescence signal for one GFP molecule. This method also minimizes measurement errors.

To test system linearity and accuracy, the metaphase and anaphasetelophase signals were evaluated for three strains expressing Nuf2p-GFP, Ndc80p-GFP or Nuf2p-GFP + Ndc80p-GFP. These measurements confirmed the 1:1 stoichiometry of Ndc80p and Nuf2p in the NDC80 complex ${ }^{7}$. As expected, the Nuf2-GFP + Ndc80p-GFP signal was twice the signal for either Nuf2p-GFP or Ndc80p-GFP alone (Fig. 3a). The variation in protein number per kinetochore is given by the s.d. of the average signal when the contribution of experimental errors is minimal. As the signal and the background can both be measured accurately in anaphase-telophase cells, the anaphase-telophase data was used for this analysis. It was found that the measured s.d. in anaphase-telophase cells was dominated by the signal loss due to spherical aberrations with increasing depth of the kinetochore cluster from the coverslip surface (Fig. 3b). Therefore, the difference in measured signal was evaluated for two kinetochore clusters in the same cell that were separated by $600 \mathrm{~nm}$ or less along the $z$ axis. The average value for this difference was approximately $10 \%$ of the average signal, suggesting that the variation in the protein number is less than one molecule per kinetochore (see Supplementary Information, Note 5). These results also apply to metaphase cells, as the protein complexes are stably anchored at the kinetochore in metaphase and anaphase.

To image single kinetochores, we used cells carrying a conditional dicentric chromosome (containing one conditional centromere in addition to the wild-type centromere ${ }^{20}$ ) and expressing Nuf2p-GFP. The induction of the conditional centromere produces one or two lagging chromatids in mid-anaphase, with their kinetochores visible as separated fluorescence foci along the spindle axis (Fig. 3c). The ratio of signal for the nearest kinetochore cluster to the signal from these foci was found to be $16: 1 \pm 2$, thus verifying that these foci are single kinetochores. These measurements demonstrate that accurate measurement of as few as seven closely clustered GFP molecules is possible in vivo (seven being the anaphase number of Nuf2p molecules per kinetochore; see Table 1). More importantly, these measurements validate our method of calculating the average number of proteins per kinetochore based on the cumulative fluorescence of 16 kinetochores in a cluster.

Table 1 lists the observed counts for the average protein number per kinetochore for representative proteins from each protein complex. A possible arrangement of the essential structural protein complexes at the budding yeast kinetochore in metaphase, based on these protein numbers, and the structures of the DAM-DASH complex and the NDC80 complex $^{7-9,21}$, is shown in Fig. 4. The protein linkage between the CEN DNA and a microtubule plus-end begins with the centromeric nucleosome incorporating two Cse4p molecules. Next is the CBF3 complex that incorporates one dimer each of Ndc10p and Cep3p. The counts for Ndc10p and Cep3p (four and two, respectively; see Supplementary Information, Note 6) together show that there is only one $\mathrm{CBF} 3$ complex per kinetochore ${ }^{22}$. It is likely that the extra Ndc10p dimer binds to the CDE II region of CEN DNA, independent of its inclusion in the CBF3 complex, as suggested by in vitro experiements ${ }^{23}$. The count for Cep3p also supports the inclusion of only two Cse $4 p$ molecules per kinetochore. The average fluorescence signal for Mif2p shows that each kinetochore has at least one Mif2p molecule, although some kinetochores carry two. Members of the COMA complex show interactions with Cse4p in two-hybrid assays ${ }^{4,24}$, along with a genetic interaction between Ctf19p and the amino $(\mathrm{N})$ terminus of Cse $4 \mathrm{p}^{24}$. The low copy number (1-2) for these protein complexes supports the presence of a network of inner kinetochore proteins ${ }^{4}$, suggested by genetic and biochemical interactions between members of these protein complexes. 
a

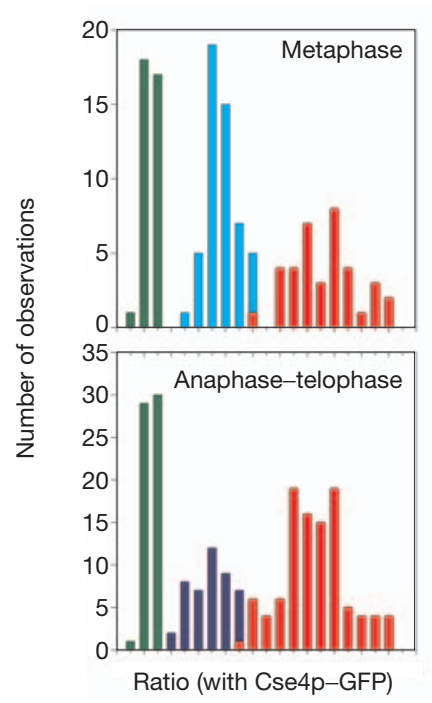

b

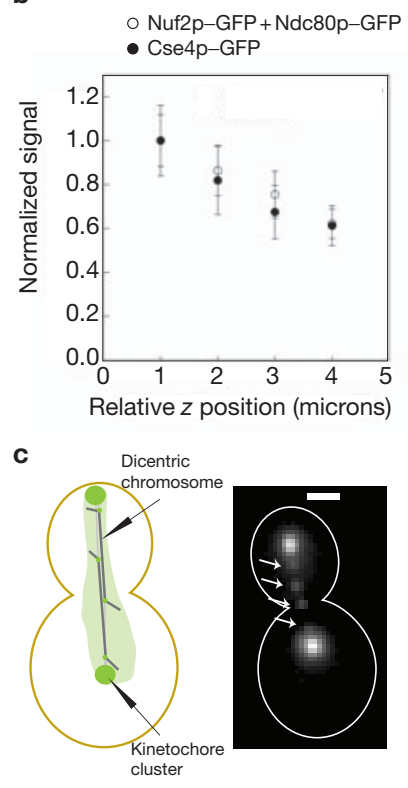

Figure 3 Linearity and sensitivity of the measurement technique. (a) A frequency histogram for signal measurements in metaphase and anaphase-telophase for Cse4p-GFP (green), Nuf2p-GFP (cyan), Ndc80pGFP (blue) and Nuf2p-GFP + Ndc80p-GFP (red). The proportional increase in the fluorescence intensity for Nuf2p-GFP + Ndc80p-GFP also demonstrates that the proximity of fluorophores does not detectably affect their fluorescence. (b) Normalized signal plotted as a function of the relative $z$ coordinate of the kinetochore clusters of Cse4p-GFP and Nuf2pGFP + Ndc80p-GFP. The error bars represent the s.d. of the mean signal value in each bin. (c) Four lagging kinetochores (arrows, Nuf2p-GFP) on the two dicentric chromosomes in a mid-anaphase cell. The chromatin between the two centromeres is $40 \mathrm{~kb}$ long. The scale bar represents $2 \mu \mathrm{m}$.

Five molecules of Spc105p were found. This protein immunoprecipitates with members of the MIND complex ${ }^{5}$, of which there are six or seven copies. The number of Mtwlp molecules is also close to the number of NDC80 complex molecules (eight). In vertebrate cells, the NDC80 complex is arranged with the Ndc80 N-terminal domain within the outer kinetochore, where microtubule plus ends are located ${ }^{15}$. Although a link between the NDC80 and the DAM-DASH complexes has not been directly established, there is indirect evidence for interactions between these complexes in the form of in vitro binding between $\mathrm{Ndc80p}$ and Dam $1 \mathrm{p}^{25}$, and two-hybrid interactions between Ndc80p and DAM-DASH complex members (Dam1p and Spc19p) ${ }^{26}$. Moreover, both of these complexes are necessary for end-on microtubule attachments. The two-headed N-termini of the NDC80 molecules are therefore positioned symmetrically around an oligomeric DAM-DASH complex ring in Fig. 4.

The metaphase count for the DAM-DASH complex (16-20 copies) reported here agrees well with the number of molecules required to form one DAM-DASH ring around a microtubule in vitro ${ }^{8}$. Biochemical estimation of the number of DAM-DASH complexes in a cell also suggests that there are not many more molecules than those needed to form one ring per kinetochore microtubule ${ }^{9}$. Because DAM-DASH is a microtubule associated protein complex, the DAM-DASH complex may also localize along the microtubule outside of the kinetochore. However, the high stability of this complex within a kinetochore cluster, in comparison with the rapid tubulin turnover within spindle microtubules (half life $=60 \mathrm{~s})^{27}$, suggests that most of the DAM-DASH complex molecules
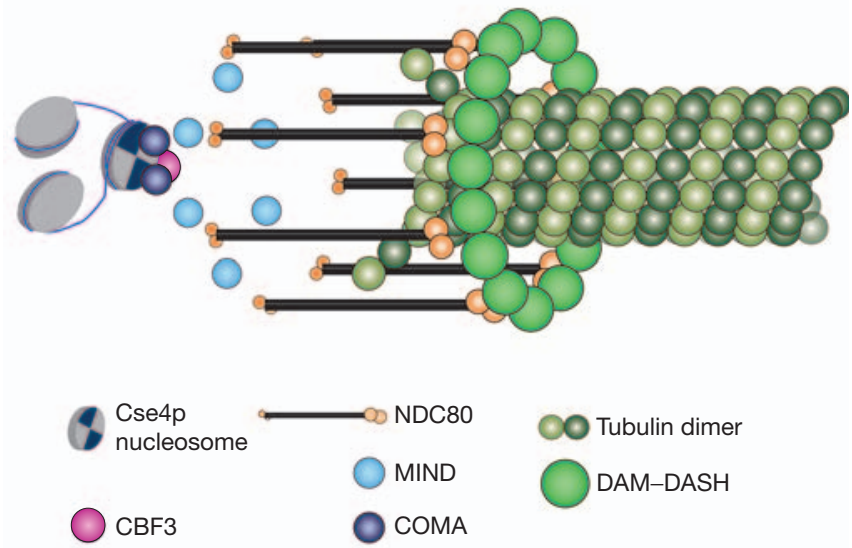

Figure $4 \mathrm{~A}$ schematic representation of the possible architecture of a kinetochore-microtubule attachment site in metaphase. This structure is based on the protein counts reported here, and on the in vitro structures of the DAM-DASH and the NDC80 complex. It depicts only five essential kinetochore protein complexes.

must bind stably at the kinetochores (see Supplementary Information, Note 7). The metaphase and late anaphase-telophase counts for all the kinetochore complexes are similar, with the exception of DAM-DASH and Ndc10p, both of which also localize to the spindle in anaphase (Fig. 1a). Interestingly, the copy numbers for the non-essential proteins ${ }^{11}$ (Ctf3p, Chl4p and Nkp2p) show that these complexes exist in minimal copy number (one per kinetochore). Preliminary measurements show that the metaphase signal for these proteins is similar to their respective anaphase-telophase signal.

This study demonstrates that the kinetochore-microtubule attachment site is built from a low and standardized number of copies of structural protein complexes. Previous studies of the structure of vertebrate kinetochores with multiple microtubule attachment sites indicate that it is constructed from a number of identical units spread along the centromere $^{28}$. This repeat-subunit structure of the kinetochore may be attained by replicating individual microtubule attachment sites, similar to the one at the budding yeast kinetochore. The molecular counts allow us to visualize possible arrangements of the structural proteins within the kinetochore. Kinetochore structure influences its function in microtubule polymerization-depolymerization-coupled force generation, in regulation of attachment stability based on mechanical cues such as tension, and in communicating attachment status to the spindle-assembly checkpoint. The combination of previous biochemical and genetic analyses with the molecular counts for structural proteins reported here provides the foundation for building a mechanistic model of these kinetochore functions.

\section{METHODS}

Yeast strains and growth conditions. All strains were constructed in the YEF473A background unless otherwise noted (see Supplementary Information, Table S5). GFP fusions were made by PCR amplification of a GFP-KAN ${ }^{r}$ cassette (from pFA6a-GFP(S65T) KAN ${ }^{r}$ MX6) flanked with 60 base pairs of homology to the site of integration at the $3^{\prime}$ end of the gene ${ }^{29}$. Cells were grown in YPD at $25^{\circ} \mathrm{C}$ to mid-log phase before imaging. For induction of the conditional centromere on the dicentric chromosome, a mid-log phase culture grown on galactose media was shifted to glucose media for $2 \mathrm{~h}$ before imaging. For imaging, cells were suspended in filter sterile synthetic dextrose media, and immobilized on standard glass coverslips coated with $0.5 \mathrm{mg} \mathrm{ml}^{-1}$ of concanavalin A (cat. no. C7275, Sigma). 
Image acquisition and data analysis. Image acquisition was carried out on an Eclipse TE2000-U (Nikon, Melville, NY) microscope with a 1.4 NA, 100× DIC oil immersion lens and the standard yEGFP filter set from Chroma (Rockingham, VT). Images were acquired with an ORCA ER cooled CCD camera (Hamamatsu Photonics, Bridgewater, NY) with $2 \times 2$ binning and using MetaMorph (Molecular Devices, Downingtown, PA) as the image acquisition software. A $300 \times 300$ pixelwide region in the middle of the field of view of the microscope was acquired to minimize the non-uniformities in the illumination field. Twenty one $z$ sections were acquired through each cell by stepping the stage in $200 \mathrm{~nm}$ steps with respect to the objective. A $400 \mathrm{~ms}$ integration time was used for all the measurements. Cells belonging to the two strains (the protein of interest and Cse4p-GFP) were differentiated based on significant differences in the fluorescence of their kinetochore clusters. This was not possible in the case of Mif2p-GFP and Ctf19p-GFP when using Cse4p-GFP in the reference strain. Therefore, a reference strain containing Nuf2p-GFP was used for these two proteins.

Data analysis was carried out in MatLAB (Mathworks Inc., Natick, MA) using a custom written graphical user interface. The plane with the maximum intensity pixel was found in a $10 \times 10$ pixel user-selected region in the $21 z$-plane stack. In this plane, the signal was computed by integrating the signal intensity in a $5 \times 5$ pixel square centred on the maximum intensity pixel for telophase measurements. A $6 \times 6$-pixel square was used for metaphase measurements because of the larger size of the signal. For anaphase-telophase cells, a larger concentric box of the appropriate dimension was used to calculate the background. The proximity of the two kinetochore clusters in metaphase cells prevented the use of this method. Therefore, the background region was chosen manually by drawing a $6 \times 6$-pixel box in the vicinity of the spindle inside the cell.

FRAP measurements. FRAP measurements were carried out on metaphase and anaphase cells expressing Ndc10p-GFP, Ctf19p-GFP, Mtw1p-GFP, Nuf2p-GFP and Ask1-GFP as previously described ${ }^{30}$.

Note: Supplementary Information is available on the Nature Cell Biology website.

\section{ACKNOWLEDGEMENTS}

We thank A. Hunt, D. Odde, S. Inoué, and members of the Salmon and Bloom laboratory for helpful comments on the manuscript. This work was supported by National Institutes of Health (NIH) grants to K.S.B. (GM32238), and to E.D.S. (GM24364 and GM60678).

\section{COMPETING FINANCIAL INTERESTS}

The authors declare that they have no competing financial interests.

Published online at http://www.nature.com/naturecellbiology/

Reprints and permissions information is available online at http://npg.nature.com/ reprintsandpermissions/

1. Meluh, P. B., Yang, P., Glowczewski, L., Koshland, D. \& Smith, M. M. Cse4p is a component of the core centromere of Saccharomyces cerevisiae. Cell 94, 607-613 (1998).

2. Meluh, P. B. \& Koshland, D. Evidence that the MIF2 gene of Saccharomyces cerevisiae encodes a centromere protein with homology to the mammalian centromere protein CENP-C. Mol. Biol. Cell 6, 793-807 (1995).

3. Espelin, C. W., Kaplan, K. B. \& Sorger, P. K. Probing the architecture of a simple kinetochore using DNA-protein crosslinking. J. Cell Biol. 139, 1383-1396 (1997).
4. Ortiz, J., Stemmann, O., Rank, S. \& Lechner, J. A putative protein complex consisting of $\mathrm{Ctf19}, \mathrm{Mcm} 21$, and $0 \mathrm{kp} 1$ represents a missing link in the budding yeast kinetochore. Genes Dev. 13, 1140-1155 (1999).

5. Nekrasov, V. S., Smith, M. A., Peak-Chew, S. \& Kilmartin, J. V. Interactions between centromere complexes in Saccharomyces cerevisiae. Mol. Biol. Cell 14, 4931-4946 (2003).

6. Euskirchen, G. M. Nnf1p, Dsn1p, Mtw1p, and Nsl1p: a new group of proteins important for chromosome segregation in Saccharomyces cerevisiae. Eukaryot. Cell 1, 229-240 (2002).

7. Wei, R. R., Sorger, P. K. \& Harrison, S. C. Molecular organization of the Ndc80 complex, an essential kinetochore component. Proc. Natl Acad. Sci. USA 102, 5363-5367 (2005).

8. Westermann, S. et al. The Dam1 kinetochore ring complex moves processively on depolymerizing microtubule ends. Nature 440, 565-569 (2006).

9. Miranda, J. J., De Wulf, P., Sorger, P. K. \& Harrison, S. C. The yeast DASH complex forms closed rings on microtubules. Nature Struct. Mol. Biol. 12, 138-143 (2005).

10. Chan, G. K., Liu, S. T. \& Yen, T. J. Kinetochore structure and function. Trends Cell Biol. 15, 589-598 (2005).

11. McAinsh, A. D., Tytell, J. D. \& Sorger, P. K. Structure, function, and regulation of budding yeast kinetochores. Annu. Rev. Cell Dev. Biol. 19, 519-539 (2003).

12. Pearson, C. G., Maddox, P. S., Salmon, E. D. \& Bloom, K. Budding yeast chromosome structure and dynamics during mitosis. J. Cell Biol. 152, 1255-1266 (2001).

13. Collins, K. A., Furuyama, S. \& Biggins, S. Proteolysis contributes to the exclusive centromere localization of the yeast Cse4/CENP-A histone H3 variant. Curr. Biol. 14, 1968-1972 (2004).

14. De Wulf, P., McAinsh, A. D. \& Sorger, P. K. Hierarchical assembly of the budding yeast kinetochore from multiple subcomplexes. Genes Dev. 17, 2902-2921 (2003).

15. Deluca, J. G. et al. Hec1 and nuf2 are core components of the kinetochore outer plate essential for organizing microtubule attachment sites. Mol. Biol. Cell 16, 519-531 (2005).

16. Wu, J. Q. \& Pollard, T. D. Counting cytokinesis proteins globally and locally in fission yeast. Science $\mathbf{3 1 0}, \mathbf{3 1 0}-314$ (2005).

17. Pearson, C. G. et al. Stable kinetochore-microtubule attachment constrains centromere positioning in metaphase. Curr. Biol. 14, 1962-1967 (2004).

18. Nishihashi, A. et al. CENP-I is essential for centromere function in vertebrate cells. Dev. Cell 2, 463-476 (2002).

19. Hori, T., Haraguchi, T., Hiraoka, Y., Kimura, H. \& Fukagawa, T. Dynamic behavior of Nuf2-Hecl complex that localizes to the centrosome and centromere and is essential for mitotic progression in vertebrate cells. J. Cell Sci. 116, 3347-3362 (2003).

20. Yang, S. S., Yeh, E., Salmon, E. D. \& Bloom, K. Identification of a mid-anaphase checkpoint in budding yeast. J. Cell Biol. 136, 345-354 (1997).

21. Ciferri, C. et al. Architecture of the human ndc80-hec 1 complex, a critical constituent of the outer kinetochore. J. Biol. Chem. 280, 29088-29095 (2005).

22. Russell, I. D., Grancell, A. S. \& Sorger, P. K. The unstable F-box protein p58-Ctf13 forms the structural core of the CBF3 kinetochore complex. J. Cell Biol. 145, 933-950 (1999).

23. Espelin, C. W., Simons, K. T., Harrison, S. C. \& Sorger, P. K. Binding of the essential Saccharomyces cerevisiae kinetochore protein Ndc10p to CDEII. Mol. Biol. Cell 14, 4557-4568 (2003).

24. Chen, $\mathrm{Y}$. et al. The $\mathrm{N}$ terminus of the centromere $\mathrm{H3}$-like protein Cse $4 \mathrm{p}$ performs an essential function distinct from that of the histone fold domain. Mol. Cell Biol. 20, 7037-7048 (2000).

25. Shang, $C$. et al. Kinetochore protein interactions and their regulation by the Aurora kinase Ipl1p. Mol. Biol. Cell 14, 3342-3355 (2003).

26. Uetz, P. et al. A comprehensive analysis of protein-protein interactions in Saccharomyces cerevisiae. Nature 403, 623-627 (2000).

27. Maddox, P. S., Bloom, K. S. \& Salmon, E. D. The polarity and dynamics of microtubule assembly in the budding yeast Saccharomyces cerevisiae. Nature Cell Biol. 2, 36-41 (2000).

28. Zinkowski, R. P., Meyne, J \& \& Brinkley, B. R. The centromere-kinetochore complex: a repeat subunit model. J. Cell Biol. 113, 1091-1110 (1991).

29. Longtine, M. S. et al. Additional modules for versatile and economical PCR-based gene deletion and modification in Saccharomyces cerevisiae. Yeast 14, 953-961 (1998).

30. Roumanie, O. et al. Rho GTPase regulation of exocytosis in yeast is independent of GTP hydrolysis and polarization of the exocyst complex. J. Cell Biol. 170, 583-594 (2005). 


\section{Supplementary Information}

(1) FRAP data - Table S1 lists the FRAP measurements. FRAP experiments were performed as described previously ${ }^{1,2}$. The measurements have been tabulated as: the number of cells with no detectable signal recovery over the time of observation, number of cells with measurable turn-over, and the fraction of signal recovered in case fluorescence recovery was observed (in parentheses).

(2) Use of Cse4p-GFP as the reference - Cse4p-GFP is a stable, core component of the kinetochore, and gets incorporated into the centromeric nucleosome during DNA synthesis ${ }^{1}$. It was therefore used as a reference for deducing the number of metaphase and anaphase/telophase molecules for the rest of the kinetochore complexes from the ratio of the average signal intensity for Cse4p-GFP and a GFP-tagged protein of interest. Cse4p-GFP turn-over within a cluster has been shown to be extremely low in metaphase spindles, both from protein dissociation and kinetochore movement from one spindle half to the other ${ }^{1}$. We verified the stability of Cse $4 p$ from metaphase to anaphase/telophase, by comparing the Cse4p-GFP signal in the respective cell phases. The measured ratio for Cse4p-GFP signal (metaphase/anaphase) was $1.07 \pm 0.01$ (based on 3 experiments). The slightly higher signal in metaphase is the result of the geometrical differences between metaphase and anaphase/telophase spindles. While the centre of the metaphase kinetochore clusters is rarely separated by more than $3 \mathrm{Z}$ planes (with $200 \mathrm{~nm}$ increments between steps), the centres of the kinetochore clusters in the longer anaphase/telophase spindles can be separated by as many as 10 $\mathrm{Z}$ planes because of the long length of the spindle. By limiting the mean signal to the 
measurements from the first $10 \mathrm{Z}$ planes of each stack, we found the metaphase to anaphase/telophase ratio to be $1.0 \pm 0.07$. The difference in the average signal value for the entire metaphase and anaphase/telophase data sets is also statistically insignificant (t-tests assuming unequal variance yield two-sided p-values of 0.12 , 0.15 , and 0.27$)$.

(3) Metaphase intensity distribution in $\mathbf{Z}$ for Ask1p-GFP - Characterization of the intensity distribution for a kinetochore cluster along the $\mathrm{Z}$ axis allows us to calculate the step-size dependent error in imaging the maxima in the intensity distribution. With a $200 \mathrm{~nm}$ distance (step-size) between successive images, the maximum error will occur when the two images are acquired at $100 \mathrm{~nm}$ on either side of the intensity maxima. With the observed intensity distribution shown in Fig. S1, this maximum error will result in an approximately $8 \%$ underestimation of the actual value. The average error will result in a $4 \%$ underestimation of the measured signal. Fig. S1 shows the average intensity distribution in Z (3 cells) for Nuf2p-GFP in anaphase/telophase (open circles). Measurements for Ask1p-GFP in metaphase are also shown (average of 2 cells, filled circles). To avoid contamination of the intensity distribution of a metaphase kinetochore cluster due to the fluorescence of the sister kinetochore cluster in the other spindle half, one of the kinetochore clusters in the cell was first bleached before carrying out the measurements on the other cluster. As seen from Fig. 2c in the main text and Fig. S1, the intensity distribution for a kinetochore cluster in both metaphase and anaphase/telophase can be approximated by that for a $200 \mathrm{~nm}$ fluorescent bead (Fig. 2c in the main text). This is consistent with the geometry of the budding yeast spindle. 


\section{(4) Error arising from the alignment of a pixel array with an imaged kinetochore}

cluster - Integration of the imaged intensity distribution with the pixel array of a CCD camera introduces a measurement error that depends on the alignment of the centre of the spot with a pixel in the pixel array. For telophase cells, a 5x5 pixel array was used to cover the spot $(143 \times 4 \mathrm{~nm})$. For anaphase/telophase measurements, the maximum intensity pixel was assigned to be the central pixel $(3,3)$ in the $5 \times 5$ pixel box. The maxima of the imaged intensity distribution will rarely align with the centre of one of the pixels introducing an error. The magnitude of this error can be estimated by considering a one dimensional Gaussian intensity distribution. The error will be minimized when the maximum of the Gaussian distribution falls exactly at the centre of a pixel of the CCD array, whereas the maximum error will occur when the maximum of the intensity distribution falls at the edge of two pixels (illustrated in Fig. S2). In reality, the alignment of the maximum of the PSF with a pixel will be uniformly distributed between these two extremes. The average error in the integrated signal will be $\sim 2 \%$ of the actual signal. The metaphase spot measurements were done using a 6x6 pixel array. The signal measurement box was drawn by assigning the maximum pixel of the image to pixel $(4,4)$. This introduces a constant error in measurement on top of a variable error similar to the case above. The error amounts to a $9 \%$ underestimation of the total signal in the worst case (when the maxima of the Gaussian curve aligns with the edge of the $(4,4)$ pixel), and a best case error of only $5 \%$ (when the maxima aligns with the centre of a pixel). The average error in this case will result in a $7 \%$ underestimation. 
It should be noted that this estimation was done for a 1-D Gaussian curve in the absence of any noise (background and shot noise). The magnitude of error is proportional to the magnitude of the signal, and its effect will be minimized in a ratio of two averaged intensity values.

(5) True signal variance - The distance of the kinetochore cluster away from the coverslip strongly affects the signal magnitude due to spherical aberrations that increase with depth (Fig. $3 b$ in the main text). This effect does not depend on the absolute magnitude of the signal, and thus does not distort the ratio of two fluorescence signals. However, the resultant variation of the signal about the mean signal masks information about the variation in the protein number.

Table S2 lists the mean and standard deviation for three different strains spanning the range of signals measured in this study. We compared the difference in the signal values for the two kinetochore clusters from the same cell that were separated by a $\mathrm{Z}$ distance of $400 \mathrm{~nm}$ or less. As can be seen from Table S2, the difference in measured intensity values for these kinetochore clusters is small as compared to the total signal. The standard error of the mean fluorescence value based on this difference is also very small. It can be stated in terms of the number of GFP molecules, by using the average Cse4p-GFP signal (1945 counts for 32 GFP molecules at 16 kinetochores -> 60 counts per GFP molecule). Thus, the difference between two kinetochore clusters in the same cell for Cse4p is 4 GFP molecules out of 32, while that for Ndc80pGFP+Nuf2p-GFP is 20 GFP molecules out of 256. It should also be noted that the standard deviation roughly scales with the mean. This translates into a variation of less than one molecule per kinetochore for each protein. 
Because of the suitable spindle geometry of late anaphase/telophase cells, it is possible to accurately measure both signal and background. The geometry of the metaphase spindles forces manual selection of the background region, introducing an additional source of variance in the signal, which is difficult to quantify. The turnover of all the complexes at the kinetochore is low in metaphase and in telophase. Therefore, the protein number variance in metaphase cells will also be similarly low.

(6) High background in cells expressing Cep3p-GFP -The high, inhomogeneous background in cells expressing Cep3p-GFP (Fig. S3) increased the measurement errors in metaphase for this protein. Both Cep3p and Ndc10p also bind spindle MTs in anaphase (Localization for Ndc10p is shown in Fig. 1a). Our measurements for the copy number in anaphase show a decrease in the number of both Cep3p and Ndc10p by the same fraction (a decrease in the ratio from $1.9 \pm 0.2$ to $1.3 \pm 0.01$ for Ndc10p and from $0.9 \pm 0.2$ to $0.6 \pm 0.01$ for Cep3p - a 1.5-fold decrease in the protein number from metaphase to anaphase in either case).

(7) Contribution of Ask1p localized to the spindle - The DAM/DASH complex localizes to kinetochores as well as spindle/interpolar MTs in anaphase. Since it is a MT-associated protein, it may localize to MTs outside of the kinetochore in metaphase. Such DAM/DASH complex molecules not localized to the kinetochore will inflate the measured signal. It is difficult to estimate the amount of DAM/DASH bound to the MTs outside of the kinetochore in metaphase cells due to the near subdiffraction size of the metaphase half-spindle. We used the fluorescence signal from Ask1p-GFP bound to the anaphase spindles to gauge the magnitude of the signal 
contributed by Ask1p-GFP bound to MTs outside of the kinetochore. To estimate the contribution of Ask1p-GFP bound along a MT, we averaged the intensity value over the in-focus sections of the spindle from several cells with an 8 pixel wide line. Each pixel in the kinetochore cluster spot was then assumed to have this average value as the contribution of DAM/DASH bound to non-kinetochore regions of the MTs. Therefore, the average pixel value multiplied by 36 (the area of a $6 x 6$ pixel box used for metaphase signal measurements) was subtracted from the average signal value for the DAM/DASH complex. This calculation reduces the measured number of DAM/DASH complex molecules by 3 or 4 . This exercise demonstrates that even after the application of this correction, there is a sufficient number (16 copies) of DAM/DASH complex molecules at the kinetochore to form one ring. The distribution of Ask1p-GFP fluorescence with respect to the fluorescence for the spindle pole bodies (Spc29-RFP) resembles the distribution of other kinetochore proteins (Fig. S4). This further suggests that most of the Ask1p-GFP remains concentrated at the kinetochores, and not on the spindle MTs.

(8) GFP response to excitation intensity - Fig. S5 shows the GFP emission response to excitation intensities. This behavior has been verified for single GFP molecules in vitro ${ }^{3}$. The data has been fitted to an equation of the form ${ }^{3}$ :

Signal $=$ constant $/(1+$ Saturation Intensity/Intensity $)$

Our protein number measurements were carried out using the highest intensity in Fig. S5 (indicated by the dotted line), which falls in the non-linear response range for the GFP molecules. The non-linear behavior does not affect ratio measurements, as the total signal is the sum of the signal response from each of the individual GFP 
molecules in the cluster to the illumination intensity. The non-linear behavior provides some protection against small fluctuations in the epi-illumination due to fluctuations in the arc lamp intensity. Fluorophore bleaching at this illumination intensity and exposure time is minimal ( 1\%). Fig. S5 also displays the linear behavior of the filters and the rest of the microscope optical system. The data was obtained by imaging $200 \mathrm{~nm}$ green fluorescent beads at a much lower excitation intensity $(\sim 0.03 \mathrm{x})$ and integration time $(50 \mathrm{~ms})$.

\section{(9) Variation in the Cse4p-GFP mean signal due to variations in excitation intensity}

- The mean signal value for Cse4p-GFP was found to be different for each experiment (Fig. S6). This is most likely due to the changes in the excitation intensity resulting from the differences in the alignment of the arc lamp with respect to the specimen plane (which was done manually), and changes in the arc lamp itself over time. This change in the mean signal value demonstrates the importance of ratio measurements as opposed to relying on the absolute fluorescence signal of a single GFP molecule.

(10) Table S3 provides the raw data for five different proteins from an experiment. Table S4 provides the list of strains used in this study.

\section{(11) Equipment and Settings -}

Microscope - Nikon Eclipse-TE2000U, 1.4 NA, 100x DIC oil immersion objective.

Filter set - Standard GFP filter set from Chroma. 
Camera - Orca ER (Hamamatsu) cooled CCD camera. 12-bit images with 2x2 binning (1 pixel 133 nm) acquired over the central 300x300 pixels of the CCD chip. Acquisition time - $400 \mathrm{~ms}$ for each frame in a 21 frame stack along the Z-direction for each microscope field.

Image acquisition software - MetaMorph 6.1 (Molecular Devices, Downingtown, PA).

Imaging conditions - Cells grown at $25^{\circ} \mathrm{C}$ in YPD to mid-log phase, re-suspended in SD complete media, and spread on coverslips coated with $0.5 \mathrm{mg} / \mathrm{ml}$ of Concanavalin A (Sigma cat. \# C7275). Microscopy was done at room temperature.

Image Analysis - Image analysis was carried out with custom written software in MatLAB (Mathworks, Natick, MA) on raw image stacks.

Displayed Images - All the images were acquired with 2x2 binning of the CCD pixel array. The montage in fig. 1a showing metaphase and anaphase spindles was prepared from a representative image for each GFP-tagged protein acquired with $400 \mathrm{~ms}$ acquisition time. The intensity range displayed is the same for the metaphase and anaphase panels. The axial intensity distribution for a kinetochore cluster shown in Fig. 2a was reconstructed from 21 planes spaced $200 \mathrm{~nm}$ apart using the 3-D reconstruction tool in Metamorph. The average background intensity was also subtracted from the image. Fig. $3 \mathrm{c}$ is the maximum intensity projection of five planes spaced $200 \mathrm{~nm}$ apart. The gamma value for this image was adjusted so that both, single kinetochores and the kinetochore cluster are clearly visible in the image. 


\begin{tabular}{|c|c|c|}
\hline Protein & Metaphase & Anaphase \\
\hline Ndc10p & $12 / 0$ & $9 / 0$ \\
\hline Ctf19p & $8 / 1(18 \%)$ & $8 / 0$ \\
\hline Mtw1p & $7 / 1(16 \%)$ & $6 / 1(7 \%)$ \\
\hline Nuf2p & $9 / 0$ & $9 / 1(9.2 \%)$ \\
\hline Ask1p & $10 / 0$ & $6 / 0$ \\
\hline
\end{tabular}

Table S1

\begin{tabular}{|l|c|c|c|}
\hline & Cse4p-GFP & Nuf2p-GFP & $\begin{array}{c}\text { Nuf2p-GFP + } \\
\text { Ndc80p-GFP }\end{array}$ \\
\hline \multicolumn{4}{|c|}{ Complete Data set } \\
\hline N (clusters) & 90 & 104 & 104 \\
\hline Average & 1945 & 6796 & 15523 \\
\hline std. dev. & 428 & 1987 & 2773 \\
\hline \multicolumn{3}{|c|}{ Clusters separated by less than 200 nm } \\
\hline N (cells) & 21 & 13 & 23 \\
\hline $\begin{array}{l}\text { Average } \\
\text { difference }\end{array}$ & 240 & 840 & 1195 \\
\hline $\begin{array}{l}\text { SEM based } \\
\text { on avg. } \\
\text { difference }\end{array}$ & 45 & 232 & 249 \\
\hline
\end{tabular}

Table S2 


\begin{tabular}{|c|c|c|c|c|c|c|c|c|c|c|c|}
\hline \multicolumn{12}{|c|}{ TELOPHASE } \\
\hline Protein & $\mathbf{N}$ & sigMean & sigDev & bkgMean & bkgDev & z1Mean & z1Std & N1 & z2Mean & Z2Std & N2 \\
\hline $\begin{array}{l}\text { Cse4p-GFP } \\
\text { Ndc10p- }\end{array}$ & 90 & 1945 & 429 & 6550 & 430 & 2215 & 663 & 17 & 2021 & 289 & 44 \\
\hline & 64 & 2550 & 677 & 7263 & 464 & 3651 & 489 & 7 & 2775 & 360 & 24 \\
\hline $\begin{array}{c}\text { GFP } \\
\text { Mtw1p- }\end{array}$ & 70 & 2912 & 681 & 7314 & 463 & 3453 & 684 & 27 & 2748 & 415 & 23 \\
\hline GFP & 98 & 6258 & 1857 & 7256 & 572 & 7691 & 2045 & 14 & 6618 & 1480 & 48 \\
\hline Nuf2p-GFP & 78 & 6797 & 1988 & 7609 & 603 & 8323 & 1905 & 16 & 6886 & 1776 & 35 \\
\hline Ask1p-GFP & 64 & 5911 & 1212 & 7968 & 795 & 6803 & 1252 & 11 & 6172 & 1031 & 24 \\
\hline \multicolumn{12}{|c|}{ METAPHASE } \\
\hline Protein & $\mathbf{N}$ & sigMean & sigDev & bkgMean & bkgDev & z1Mean & z1Std & N1 & z2Mean & z2Std & N2 \\
\hline $\begin{array}{l}\text { Cse4p-GFP } \\
\text { Ndc10p- }\end{array}$ & 28 & 2419 & 529 & 10251 & 1538 & 2697 & 33 & 2 & 2148 & 574 & 10 \\
\hline $\begin{array}{l}\text { GFP } \\
\text { Ctf19p- }\end{array}$ & 34 & 4070 & 1443 & 11132 & 879 & 5336 & 1596 & 7 & 3742 & 1262 & 25 \\
\hline $\begin{array}{c}\text { GFP } \\
\text { Mtw1p- }\end{array}$ & 70 & 2912 & 681 & 7314 & 463 & 3453 & 684 & 27 & 2748 & 415 & 23 \\
\hline GFP & 22 & 6831 & 1832 & 9928 & 208 & 7531 & 897 & 4 & 6737 & 1988 & 16 \\
\hline Nuf2p-GFP & 22 & 9327 & 1608 & 10728 & 1050 & 10330 & 1753 & 6 & 8951 & 1430 & 16 \\
\hline Ask1p-GFP & 18 & 24487 & 7817 & 11319 & 1167 & 29320 & 4038 & 3 & 24036 & 8163 & 14 \\
\hline
\end{tabular}

Table S3 lists the representative data sets for each of the proteins considered in this study. It should be noted that the data sets for Cse4p-GFP and Nuf2p-GFP come from the same experiment, thus making ratio calculation meaningful for the pair.

$\mathrm{N}=$ total number of measurements

sigMean $=$ Mean Signal

sigDev $=$ standard deviation for the measured signal

bkgMean $=$ Mean background

bkgDev $=$ standard deviation for the background

$\mathrm{z} 1$ Mean $(\mathrm{z} 2 \mathrm{Mean})=$ mean signal for measurements done in the 1-5(6-10) $\mathrm{Z}$ planes

$\mathrm{z} 1 \mathrm{Std}(\mathrm{z} 2 \mathrm{Std})=$ corresponding standard deviation

$\mathrm{N} 1, \mathrm{~N} 2=$ Number of measurements for the respective subgroups 


\begin{tabular}{|c|c|c|}
\hline Strain & Genotype & Source \\
\hline KBY7006 & YEF 473a Cse4-GFP:KAN ${ }^{\mathrm{R}}$ & Bloom lab \\
\hline KBY2310 & YEF 473a Ndc10-GFP:HIS & Bloom lab \\
\hline DCB110 & YEF 473a Cep3-GFP:HIS & Bloom lab \\
\hline KBY7013 & YEF 473a Mif2-GFP:KAN ${ }^{\mathrm{R}}$ & Bloom lab \\
\hline KBY7009 & YEF 473a Ctf19-GFP:KAN ${ }^{R}$ & Bloom lab \\
\hline SWY40B & S288C MATa his3 200, leu2-3,112, ura3-52, lys2, Mtw1-GFP:KAN ${ }^{R}$ & Drubin Lab \\
\hline KBY5056 & YEF 473a Nuf2-GFP:HB ${ }^{\mathrm{R}}$ & Bloom lab \\
\hline KBY7005 & YEF 473a Ndc80-GFP:KAN ${ }^{R}$ & Bloom lab \\
\hline ICY211D & S288C MATa his3 200, leu2-3,112, ura3-52, lys2, Ask1-GFP:KAN ${ }^{R}$ & Drubin Lab \\
\hline KBY7012 & YEF 473a Ctf3-GFP:KAN ${ }^{R}$ & Bloom lab \\
\hline KBY4014 & 9c MATa, ura3, leu2, Chl4-GFP:KAN ${ }^{R}$ & Bloom lab \\
\hline KBY7016 & YEF 473a Nkp2-GFP:KAN ${ }^{R}$ & Bloom lab \\
\hline KBY7008 & YEF 473a Nuf2-GFP:HB ${ }^{\mathrm{R}}$ Ndc80-GFP: KAN ${ }^{\mathrm{R}}$ & Bloom lab \\
\hline KBY4139 & J178D His4::Gal-CEN:HB Nuf2-GFP:URA3 & Bloom lab \\
\hline
\end{tabular}

Table S4 


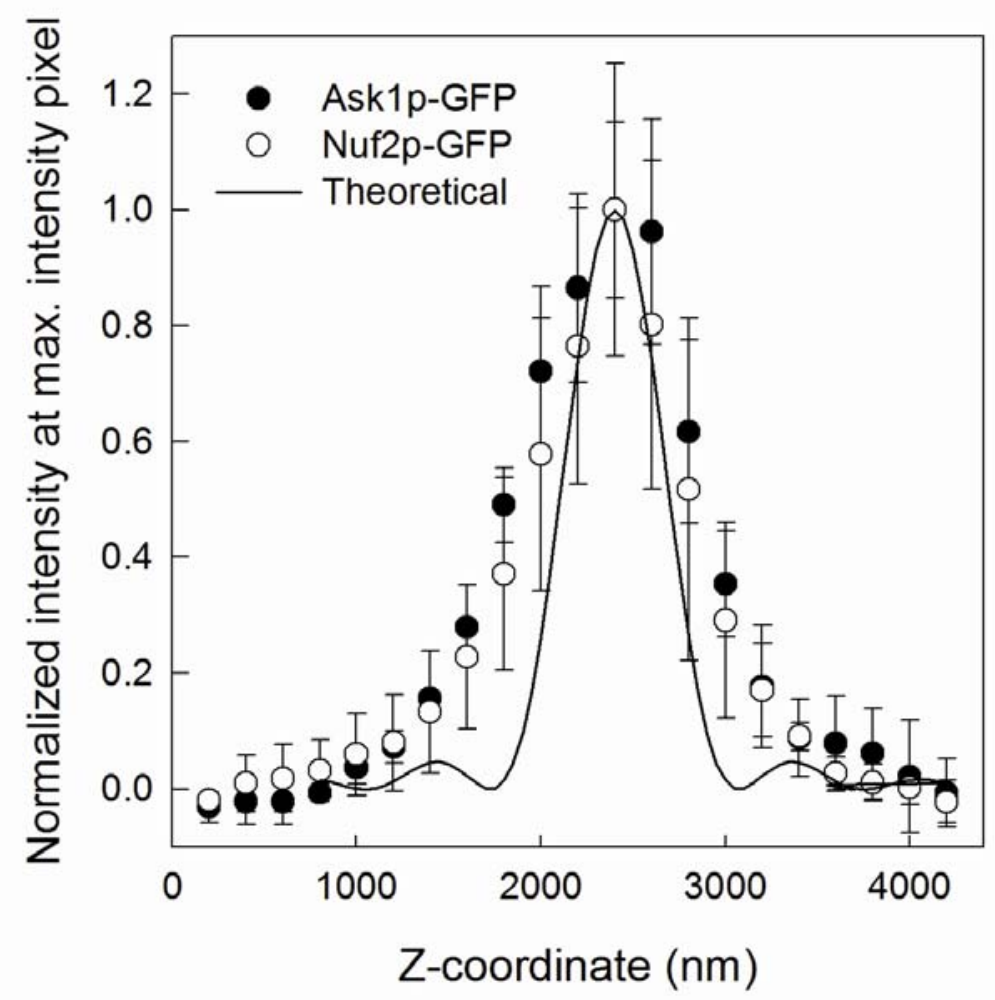

Figure S1 

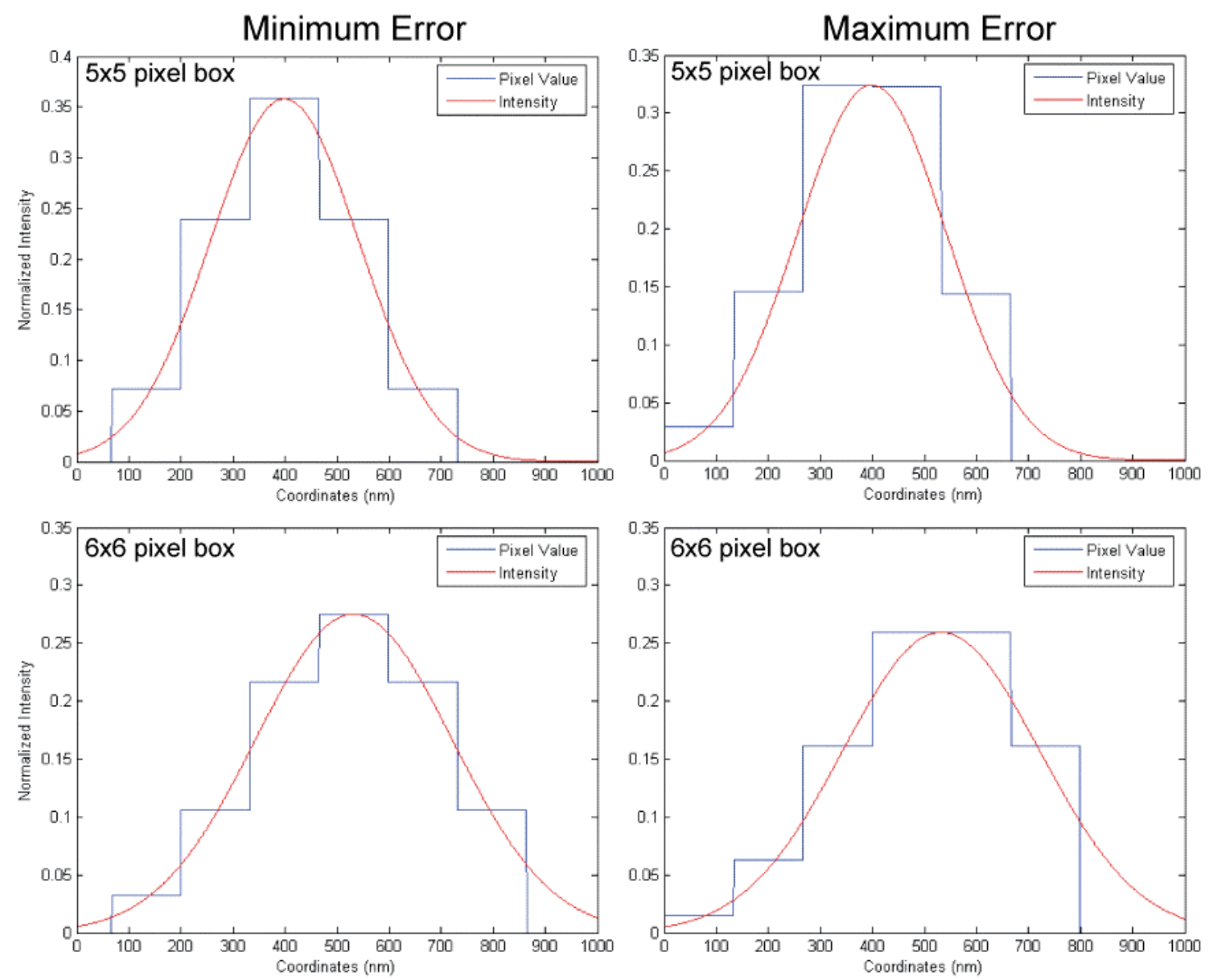

Figure S2 


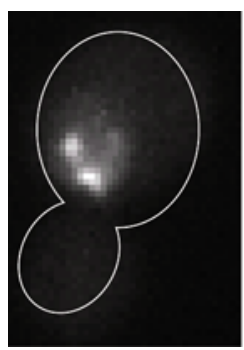

Cep3p-GFP

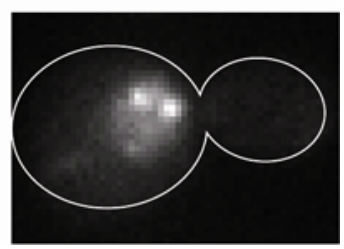

Figure S3 

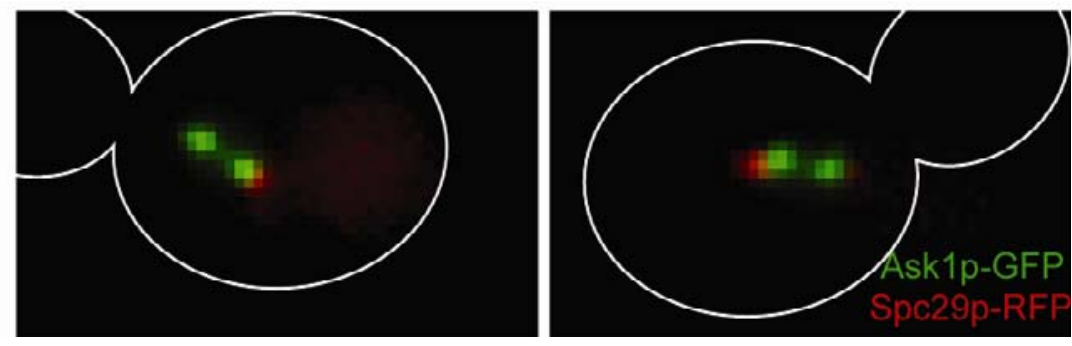

Figure S4 

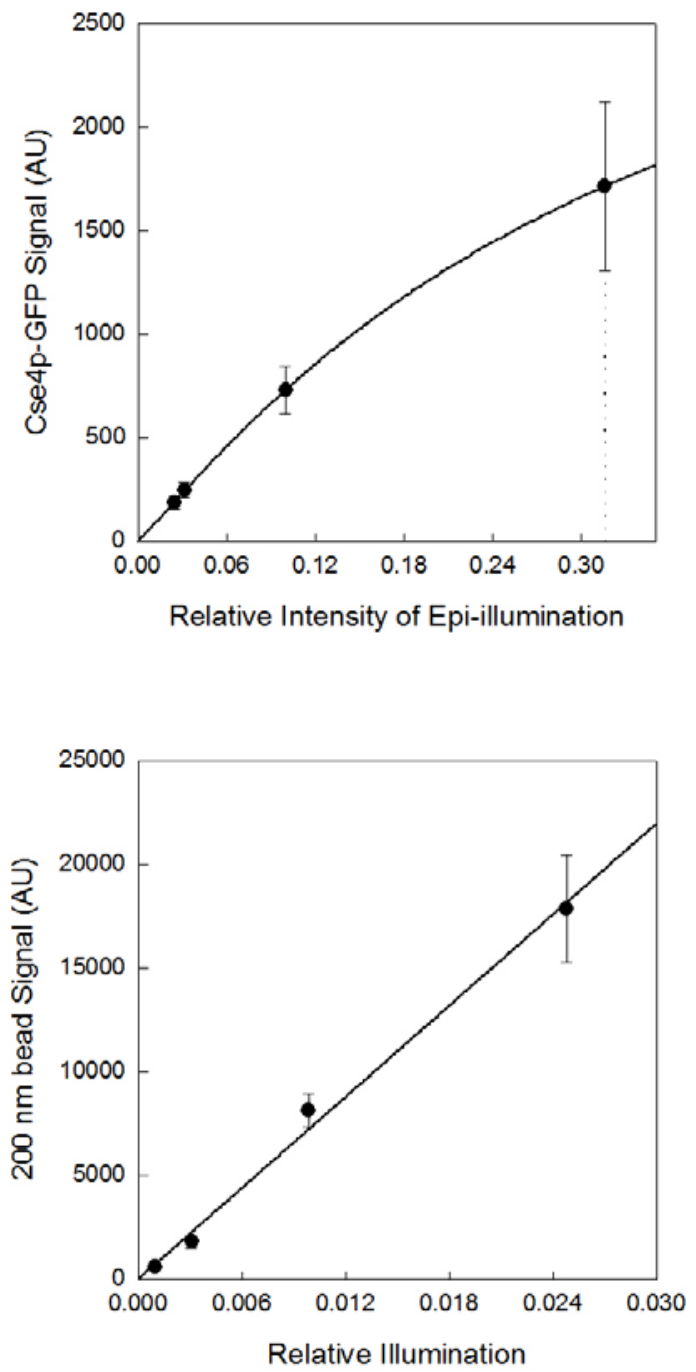

Figure S5 


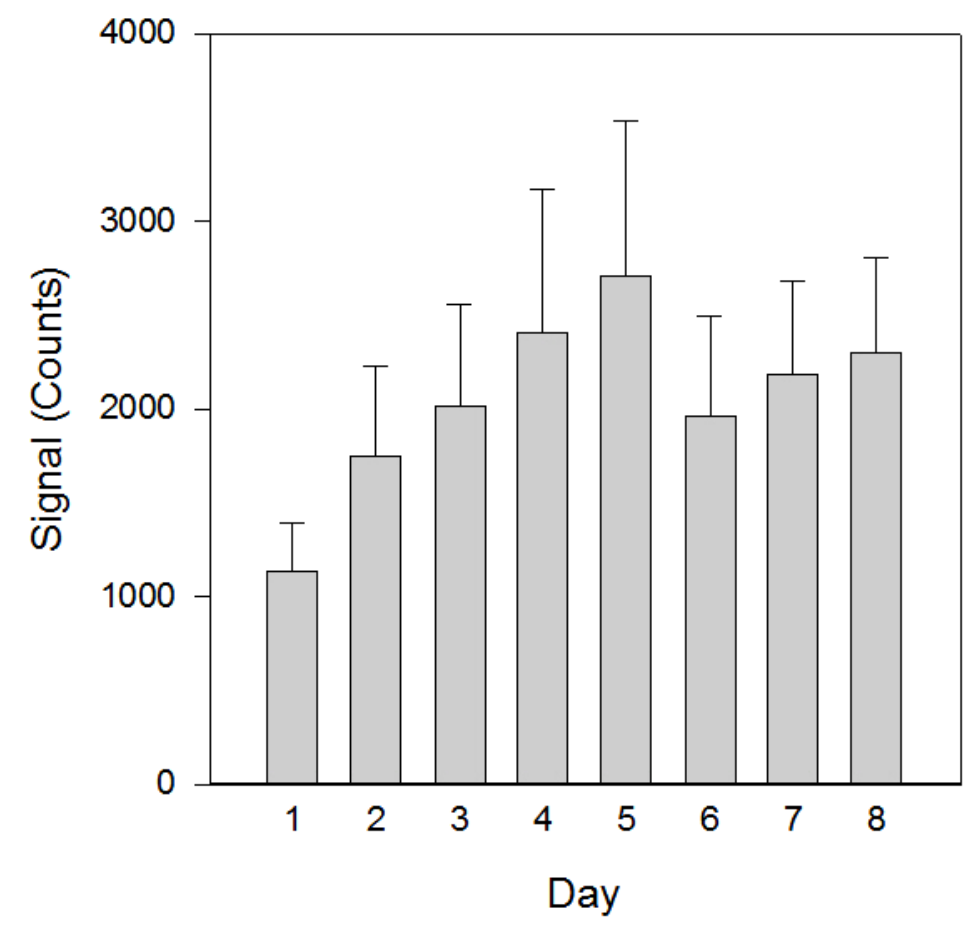

Figure S6 


\section{REFERENCES}

1. Pearson, C. G. et al. Stable kinetochore-microtubule attachment constrains centromere positioning in metaphase. Curr Biol 14, 1962-7 (2004).

2. Roumanie, O. et al. Rho GTPase regulation of exocytosis in yeast is independent of GTP hydrolysis and polarization of the exocyst complex. J Cell Biol 170, 583-94 (2005).

3. Kubitscheck, U., Kuckmann, O., Kues, T. \& Peters, R. Imaging and tracking of single GFP molecules in solution. Biophys J 78, 2170-9 (2000). 